CONGENITAL HEART DISEASE

\title{
Cardiovascular response to physical exercise in adult patients after atrial correction for transposition of the great arteries assessed with magnetic resonance imaging
}

\author{
A A W Roest, H J Lamb, E E van der Wall, H W Vliegen, J G van den Aardweg, P Kunz, A de Roos, \\ W A Helbing
}

Heart 2004;90:678-684. doi: 10.1136/hrt.2003.023499

\begin{abstract}
See end of article for authors' affiliations

Correspondence to:

$\operatorname{Dr}$ A de Roos, Department of Radiology C2-S, Leiden University Medical Centre, Albinusdreef 2, 2333 ZA Leiden, Netherlands; a.de_roos@lumc.nl
\end{abstract}

Accepted 16 October 2003

\begin{abstract}
Objective: To assess with magnetic resonance imaging (MRI) cardiovascular function in response to exercise in patients after atrial correction of transposition of the great arteries (TGA).

Methods: Cardiac function at rest and during submaximal exercise was assessed with MRI in 27 patients with TGA (mean (SD) age 26 (5) years) late (23 (2) years) after atrial correction and in 14 control participants (25 (5) years old).

Results: At rest, only right ventricular ejection fraction was significantly lower in patients than in controls (56 (7)\% v $65(7) \%, p<0.05)$. In response to exercise, increases in right ventricular end diastolic (155 (55) $\mathrm{ml}$ to $163(57) \mathrm{ml}, \mathrm{p}<0.05)$ and right ventricular end systolic volumes (70 (34) $\mathrm{ml}$ to $75(36) \mathrm{ml}$, $\mathrm{p}<0.05)$ were observed in patients. Furthermore, right and left ventricular stroke volumes and ejection fraction did not increase significantly in patients. Changes in right ventricular ejection fraction with exercise correlated with diminished exercise capacity $(r=0.43, p<0.05)$.

Conclusions: In patients with atrially corrected TGA, MRI showed an abnormal response to exercise of both systemic right and left ventricles. Exercise MRI provides a tool for close monitoring of cardiovascular function in these patients, who are at risk for late death.
\end{abstract}

$\mathrm{T}$ ransposition of the great arteries (TGA) is one of the most common types of cyanotic congenital heart disease. ${ }^{1}$ The arterial switch operation is the procedure of choice. ${ }^{23}$ Before the introduction of the arterial switch operation, TGA was corrected at the atrial level by the Mustard or Senning technique. A substantial number of patients are still alive after atrial correction of TGA and provide a challenge to clinicians.

After atrial correction, the right ventricle continues to be subjected to systemic afterload. Although the majority of patients are asymptomatic, ${ }^{4}$ reduced exercise capacity, ${ }^{5}$ cardiac dysfunction at rest $^{6}$ or in response to exercise, obstruction of the venous pathways, ${ }^{8}$ and arrhythmias ${ }^{9}$ have been reported. Systemic right ventricular failure, atrial arrhythmias, and baffle obstruction are risk factors for late death of patients after atrial correction of TGA. ${ }^{10-13}$ Furthermore, right ventricular dysfunction contributes to the occurrence of atrial arrhythmias in these patients, ${ }^{10}{ }^{12}$ stressing the need for close monitoring of the function of the systemic right ventricle.

Assessment of cardiac function during exercise may disclose cardiac dysfunction, which may not be apparent at rest. ${ }^{14}{ }^{15}$ However, non-invasive quantification of biventricular volumes and function at rest and during exercise with commonly used non-invasive techniques such as radionuclide ventriculography ${ }^{1617}$ or echocardiography ${ }^{18}{ }^{19}$ has important limitations. Magnetic resonance imaging (MRI) is an accurate and non-invasive technique to assess cardiac morphology and function at rest. ${ }^{60-22}$ Recently, MRI techniques have become available to study cardiovascular response to supine physical exercise. ${ }^{23} 24$

The purpose of the present study was to assess biventricular response to supine physical exercise with the use of MRI in patients late after atrial correction of TGA.

\section{PATIENTS AND METHODS}

Twenty seven patients with TGA (mean (SD) age 25.7 (4.6) years) corrected at the atrial level at a mean age of 2.5 (2.2) years who were scheduled to undergo cardiac MRI examination at rest for clinical follow up of cardiovascular function and who did not have contraindications for MRI examination, such as the presence of a pacemaker, arrhythmias, claustrophobia, or the inability to perform bicycle exercise, were examined and compared with 14 control participants. The controls were normal at clinical examination and had a normal ECG at rest, no history of cardiovascular disease, and no complaints. The controls were matched for age (24.8 (5.2) years), weight, height, and body surface area (table 1). TGA had been corrected by the Mustard procedure in 11 patients and by the Senning procedure in 16 patients. Sixteen patients had additional pulmonary stenosis, ventricular septal defect, or both, which is referred to as complex TGA. All patients were in New York Heart Association (NYHA) class I or II and all were in regular cardiac rhythm (25 patients were in sinus rhythm and two patients had an atrioventricular junctional rhythm). Informed consent was obtained from all participants and the medical review board of our institution approved the study.

\section{Exercise testing}

Exercise testing was performed before the MRI examination on a separate day. A graded maximal exercise test was performed in the supine position on an MRI compatible ergometer (MRI cardiac ergometer, Lode BV, Groningen, the

Abbreviations: MRI, magnetic resonance imaging; NYHA, New York Heart Association; TFEPI, turbofield echo planar imaging; TGA, transposition of the great arteries 
Table 1 Characteristics of the study population

\begin{tabular}{|c|c|c|c|c|}
\hline & Controls $(n=14)$ & Total TGA $(n=27)$ & Mustard ( $n=11)$ & Senning $(n=16)$ \\
\hline Male/female & $7 / 7$ & $15 / 12$ & $7 / 4$ & $8 / 8$ \\
\hline Age (years) & $24.8(5.2)$ & $25.7(4.6)$ & $29.2(3)$ & $23.3(3.9)^{*}$ \\
\hline Height $(m)$ & $1.78(0.06)$ & $1.73(0.09)$ & $1.72(0.11)$ & $1.74(0.08)$ \\
\hline Weight (kg) & $73.8(11)$ & $71.7(16)$ & $66.8(15)$ & $75.1(17)$ \\
\hline $\mathrm{BSA}\left(\mathrm{m}^{2}\right)$ & $1.9(0.2)$ & $1.9(0.2)$ & $1.8(0.2)$ & $1.9(0.2)$ \\
\hline Simple/complex TGA† & NA & $11 / 16$ & $7 / 4$ & $4 / 12$ \\
\hline Age at operation & NA & $2.5(2.2)$ & $2.5(1.1)$ & $2.5(2.7)$ \\
\hline Balloon septostomy & NA & 17 & 6 & 11 \\
\hline Blalock-Hanlon septostomy & NA & 9 & 6 & 3 \\
\hline
\end{tabular}

Netherlands) with continuous recording of ECG and arterial oxygen saturation. The following parameters were measured by a gas analyser (Oxycon Record, Jaeger, Höchberg, Germany) during the exercise test: total ventilation, breathing frequency, oxygen uptake, and carbon dioxide release. Ventilatory equivalents at peak exercise for carbon dioxide (ratio of maximum total ventilation to maximum carbon dioxide release) and for oxygen (ratio of maximum total ventilation to maximum oxygen uptake) were calculated. The respiratory quotient (ratio of carbon dioxide release to oxygen uptake) at peak exercise was used as an indicator of maximum effort.

\section{Magnetic resonance imaging}

The MRI examination was done with a Philips 1.5 T ACSNT15 magnetic resonance scanner (Philips Medical Systems, Best, the Netherlands) equipped with a Powertrak 6000 gradient system and a CPR-6 cardiac research software package (Philips Medical Systems). After scout views were obtained, MRI flow velocity was measured with retrospective electrocardiographic gating at rest in the pulmonary trunk, in the ascending aorta, at the level of both the mitral and tricuspid valves, and in the venous pathways, as previously described. ${ }^{25}$ At rest and during exercise, 10 consecutive slices in the left ventricular short axis orientation with a thickness of $10 \mathrm{~mm}$ and an interslice gap of $1 \mathrm{~mm}$ were used to cover the left and right ventricles from apex to base. The short axis images were obtained by a turbofield echo planar imaging (TFEPI) sequence with prospective electrocardiographic triggering. Imaging parameters were a $128 \times 40$ matrix, $420 \times 120 \mathrm{~mm}$ field of view, $30^{\circ}$ flip angle, repetition time of $14 \mathrm{~ms}$, echo time of $4.8 \mathrm{~ms}$, echo planar imaging factor of 5 , turbo factor of 2, and temporal resolution of $31 \mathrm{~ms}$. All $\mathrm{k}$ lines of one short axis slice were obtained during four heart beats. Two short axis slices were acquired sequentially during a breath hold of eight heart beats at end expiration.

Exercise was performed on the MRI compatible ergometer. The exercise level for the MRI examination was based on the workload corresponding to $60 \%$ of the maximum oxygen uptake measured during the preceding maximal exercise test. After reaching a steady heart rate, the participants performed a breath hold procedure and images were acquired within eight heart beats during a short exercise break. After acquisition of the images the participants continued cycling until a steady heart rate was again reached. This exercise protocol has previously been validated by Pedersen and colleagues, ${ }^{26}$ who used a time window of 12 heartbeats for the acquisition. Mean decrease in heart rate immediately after exercise was $4 \%$ after six heart beats and less than $14 \%$ after 11 heart beats, which was considered acceptable for the evaluation of haemodynamic changes during exercise. With our TFEPI MRI sequence we obtained two short axis slices during eight heart beats, which is within the limits used by Pedersen and colleagues. ${ }^{26}$ The breath hold procedure was repeated to acquire a total of 10 short axis slices.

\section{Data analysis}

MRI flow velocity measurements were analysed by the FLOW software package (Medis, Leiden, the Netherlands). ${ }^{27}$ Quantitative analysis of the short axis images by the MASS software package (Medis, Leiden, the Netherlands) ${ }^{28}$ allowed calculation of the following parameters of both ventricles: end diastolic volume, end systolic volume, stroke volume, and ejection fraction. In line with previous reports, ${ }^{625}$ the systemic right ventricular function of the TGA patients was compared with the right ventricular function of the control group, and the left ventricular function of the patients was compared with the left ventricular function of the controls.

Abnormal right ventricular ejection fraction at rest was defined as less than $47 \%\left(-2\right.$ SD of normal value $\left.{ }^{29}{ }^{30}\right)$.

A normal ventricular response to exercise was defined as an increase in ejection fraction of more than $5 \%{ }^{31}$ All data were expressed as mean (SD).

\section{Statistical analysis}

An unpaired Student's $t$ test was used to compare the parameters of exercise performance and cardiac function between the patients with atrially corrected TGA and the matched control group. A paired Student's $t$ test was used to compare the parameters of cardiac function obtained at rest and during the exercise protocol. Pearson correlation analysis was used to evaluate correlation between parameters. The level of significance was $p<0.05$.

\section{RESULTS}

\section{Exercise testing}

No difference in respiratory quotient at peak exercise was observed between the TGA and the control groups (table 2). All participants reached a respiratory quotient above 1.0, indicating at least near maximum effort during the graded exercise test. ${ }^{32}$ At peak exercise the TGA patients had a significantly lower oxygen uptake per kilogram bodyweight

Table 2 Results of maximal exercise testing

\begin{tabular}{lll}
\hline & Controls & TGA patients \\
\hline Respiratory quotient & $1.17(0.04)$ & $1.15(0.06)$ \\
Heart rate (beats/min) & $175(8)$ & $160(23)^{*}$ \\
Work load (W) & $231(32)$ & $156(35)^{*}$ \\
Total ventilation (l/min) & $105(22)$ & $79(21)^{*}$ \\
Breathing frequency (breaths/min) & $46(7)$ & $48(12)$ \\
Oxygen uptake $(\mathrm{l} / \mathrm{min})$ & $3.1(0.5)$ & $2.1(0.6)^{*}$ \\
Oxygen uptake per $\mathrm{kg}(\mathrm{ml} / \mathrm{min} / \mathrm{kg})$ & $42(5)$ & $29(7)^{*}$ \\
\hline${ }^{*} \mathrm{p}<0.05$ TGA patients versus healthy controls. & \\
\hline
\end{tabular}


than did the controls $(29.1(7.3) \mathrm{ml} / \mathrm{min} / \mathrm{kg} v 42.0(5.1) \mathrm{ml} /$ $\mathrm{min} / \mathrm{kg}, \mathrm{p}<0.01)$. Furthermore, peak workload of the TGA patients was lower (156 (35) W $v 231(32 \mathrm{~W}), \mathrm{p}<0.01)$. Heart rate at peak exercise was lower in the TGA group ( 160 (23) beats/min $v 175$ (8) beats/min, p < 0.05). Breathing frequency did not differ between the patients and the controls (table 2). No difference in exercise capacity was observed between patients with simple TGA and those with complex TGA or between patients who had undergone the Mustard procedure and those with the Senning procedure. The two patients who had atrioventricular junctional rhythm converted to sinus rhythm during exercise.

\section{Cardiac function at rest}

At rest, the systemic right ventricular ejection fraction was significantly lower in the patients than in controls $(56(7) \% v$ $65(7) \%, \mathrm{p}<0.05)$. However, 24 of the 27 patients $(89 \%)$ had a right ventricular ejection fraction greater than $47 \%$. The wall mass of the right ventricle was significantly higher in the patients than in the controls (112 (43) g v 4l (7) g, $\mathrm{p}<0.05)$, whereas left ventricular wall mass was significantly lower in the patients (85 (32) g $v 133$ (21) g, $\mathrm{p}<0.05$ ). All other parameters of left and right ventricular function were not significantly different between the groups (table 3).

One patient was found to have a stenosis in the pulmonary venous pathway. Minimum tricuspid valve regurgitation (6 (5)\%) was observed in 15 patients. No residual ventricular septal defect was observed in the patient group. In one patient high velocities (peak $210 \mathrm{~cm} / \mathrm{s}$ ) in the pulmonary artery suggested the presence of mild pulmonary stenosis.

Resting right ventricular ejection fraction did not correlate with exercise capacity $(r=0.1, \mathrm{p}=0.60)$ in the patients. No difference in cardiac function was observed between patients with simple TGA and those with complex TGA or between the patients who had undergone the Mustard and those who had undergone the Senning procedures.

\section{Cardiac response to supine bicycle exercise}

In response to the submaximal MRI exercise level, heart rate increased from 68 (10) beats/min to 117 (8) beats/min $(\mathrm{p}<0.05)$ in the TGA patients and from 71 (9) beats/min to 121 (14) beats/min $(p<0.05)$ in the controls. No difference in heart rate at the MRI exercise level was observed between the TGA patients and the controls.

The response of both the left and the right ventricles to exercise was analysed for all participants (fig 1) and was significantly different between the TGA group and the controls (table 3). As expected, biventricular stroke volume and ejection fraction increased significantly in the controls. In all controls biventricular ejection fraction increased more than $5 \%$. In the TGA patients stroke volume and ejection fraction of both ventricles did not change significantly in response to exercise. In only one patient systemic right ventricular ejection fraction increased more than $5 \%$ in response to exercise, and in 10 patients left ventricular ejection fraction increased more than 5\%.

In response to exercise, volume changes of both ventricles differed significantly between the TGA patients and the controls (table 3, fig 2). Systemic right ventricular end diastolic and end systolic volumes of the TGA patients increased in response to exercise (end diastolic volume: 155 (55) to 163 (57) $\mathrm{ml}, \mathrm{p}<0.05$; end systolic volume: 70 (34) $\mathrm{ml}$ to $75(36) \mathrm{ml}$ ), whereas in the controls right ventricular end diastolic volume did not change and end systolic volume decreased $(52$ (20) $\mathrm{ml}$ to 37 (16) $\mathrm{ml}$, $p<0.05)$. Left ventricular end diastolic and end systolic volumes of the TGA patients did not change significantly in response to exercise, whereas in the controls left ventricular end systolic volume decreased without a change in end diastolic volume.

In the TGA patients, systemic right ventricular ejection fraction during exercise, but not at rest, and the percentage change in right ventricular ejection fraction from rest to exercise correlated significantly with peak oxygen consumption per kilogram bodyweight (exercise right ventricular ejection fraction: $r=0.40, \mathrm{p}<0.05$; change in right ventricular ejection fraction: $r=0.43, \mathrm{p}<0.05)$.

\section{DISCUSSION}

In the present study, cardiovascular function at rest and in response to supine exercise was evaluated with MRI in patients with TGA $23(2)$ years after atrial correction and in a control group. The main finding was an abnormal response of both ventricles to exercise in the TGA patients. Despite a relatively well preserved resting ventricular function, both the right and left ventricles failed to increase stroke volume and ejection fraction in response to exercise and the systemic right ventricular end diastolic and end systolic volumes increased. The abnormal changes in right ventricular ejection fraction in response to exercise correlated with impaired exercise capacity in the patients with atrially corrected TGA.

\section{Cardiac function at rest}

At rest, the ejection fraction of the systemic right ventricle differed significantly between the controls and TGA patients

Table 3 Cardiac response to exercise

\begin{tabular}{|c|c|c|c|c|c|c|}
\hline & \multicolumn{3}{|l|}{ Controls } & \multicolumn{3}{|c|}{ TGA patients } \\
\hline & Rest & Exercise & $\%$ Change & Rest & Exercise & $\%$ Change \\
\hline Workload (W) & NA & $132(16)$ & 0 & NA & $87(20)$ & \\
\hline Heart rate (beats/min) & $67(8)$ & $122(8) \dagger$ & $85(23)$ & $68(10)$ & $112(15) \dagger$ & $68(26)^{*}$ \\
\hline \multicolumn{7}{|l|}{ Right ventricle } \\
\hline $\mathrm{EDV}(\mathrm{ml})$ & $146(24)$ & $148(26)$ & $1(4)$ & $155(55)$ & $163(57) \dagger$ & $5(8)^{*}$ \\
\hline $\mathrm{ESV}(\mathrm{ml})$ & $52(20)$ & $37(16) \dagger$ & $-30(13)$ & $70(34)$ & $75(36) t$ & $9(16)^{*}$ \\
\hline $\mathrm{SV}(\mathrm{ml})$ & $96(10)$ & $111(13) \dagger$ & $16(6)$ & $85(26)$ & $87(25)$ & $3(9)^{*}$ \\
\hline $\mathrm{EF}(\%)$ & $65(7)$ & $76(7)+$ & $16(7)$ & $56(7)^{*}$ & $55(7)$ & $-2(8)^{*}$ \\
\hline Mass (g) & $41(7)$ & NA & $\mathrm{NA}$ & $112(43)^{*}$ & NA & NA \\
\hline \multicolumn{7}{|l|}{ Left ventricle } \\
\hline EDV (ml) & $146(24)$ & $148(26)$ & $1(4)$ & $137(42)$ & $135(43)$ & $-1(9)$ \\
\hline $\mathrm{ESV}(\mathrm{ml})$ & $51(18)$ & $36(14) \dagger$ & $-30(9)$ & $50(21)$ & $47(22)$ & $-5(22)^{*}$ \\
\hline $\mathrm{SV}(\mathrm{ml})$ & $96(10)$ & $112(15) \dagger$ & $17(8)$ & $87(25)$ & $88(24)$ & $2(8)^{*}$ \\
\hline $\mathrm{EF}(\%)$ & $66(7)$ & $77(6) \dagger$ & $16(8)$ & 64 (7) & $66(7)$ & $3(9)^{*}$ \\
\hline Mass $(\mathrm{g})$ & $133(21)$ & NA & $\mathrm{NA}$ & $85(32)^{*}$ & NA & NA \\
\hline
\end{tabular}


Rest

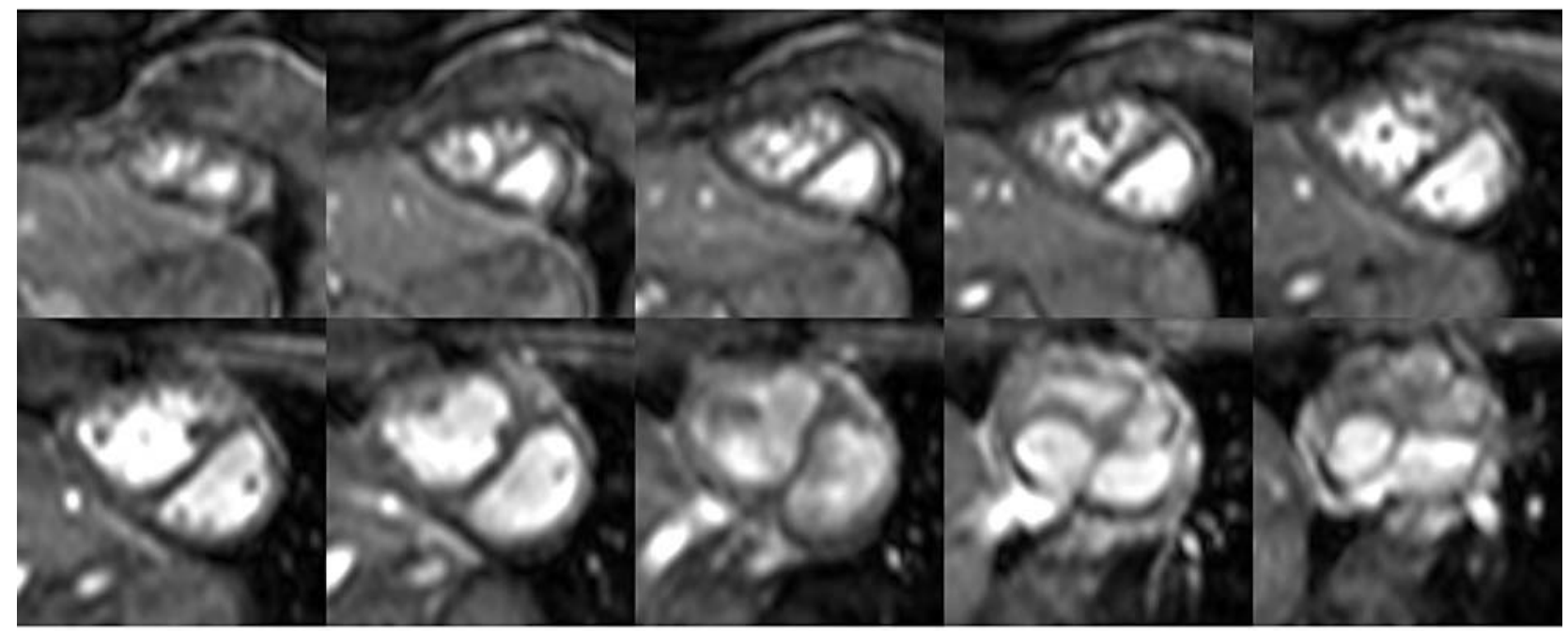

Exercise

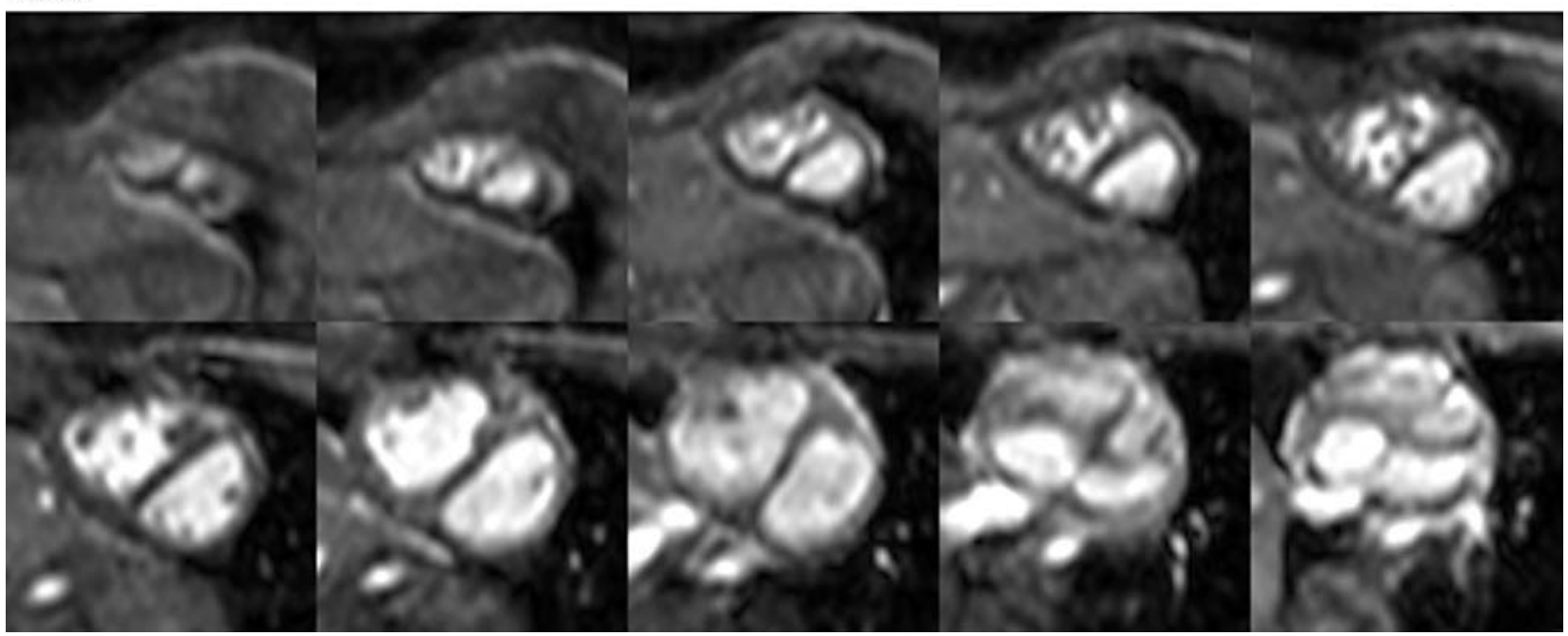

Figure 1 Short axis images at rest and during exercise at end diastole from a patient with atrially corrected transposition of the great arteries obtained with an ultrafast turbofield echo planar magnetic resonance imaging sequence. The interventricular septum is displaced towards the left ventricle, especially at end systole. Note the image quality of the images obtained at rest and during the exercise protocol.

23 (2) years after atrial correction. However, in the majority of our patients $(89 \%)$ right ventricular ejection fraction was within normal limits. ${ }^{29}{ }^{30}$ Lorenz and colleagues ${ }^{6}$ reported similar results with the use of MRI with 22 patients 16 (5) years after atrial correction of TGA. In that study, ${ }^{6}$ normal systemic right ventricular size, a mildly depressed right ventricular ejection fraction, increased right ventricular wall mass, and normal left ventricular function were found. In a longitudinal study, ${ }^{33}$ serial measurements of systemic ventricular performance showed no deterioration over time. In our study the results suggest that resting global biventricular function is relatively well preserved during follow up after atrial correction of TGA.

Quantification of tricuspid valve regurgitation showed that this was a common finding in the TGA patients, although the amount was small in most patients. The presence of tricuspid regurgitation is in concordance with earlier reports, ${ }^{11}{ }^{34} 35$ but it is unclear whether tricuspid regurgitation is a primary phenomenon or a result of right ventricular dysfunction. Tricuspid valve regurgitation may play a part in the occurrence of atrial arrhythmias and right ventricular dysfunction. Close follow up is warranted, ${ }^{12}$ which can be done accurately and non-invasively with MRI.

\section{Cardiac function in response to exercise}

To evaluate the biventricular response to exercise in the TGA patients after atrial correction we used a dedicated MRI exercise protocol. This approach has already been validated in healthy young adults ${ }^{23} 24$ and in patients after correction of tetralogy of Fallot. ${ }^{15}$ Low intraobserver and interobserver variabilities of $4(3) \%$ and $8(7) \%$, respectively, for the left ventricle and $5(4) \%$ and $9(8) \%$, respectively, for the right ventricle have been obtained..$^{23}$

Despite the well preserved systemic ventricular function of the TGA patients after atrial correction, the right ventricular ejection fraction response to exercise was abnormal in 26 of the 27 TGA patients. Mean stroke volume and the ejection fraction of both the right and left ventricles of the TGA patients did not increase in response to exercise. Similar observations for stroke volume and ejection fraction response have been reported previously when radionuclide angiography was used..$^{36-40}$ But others ${ }^{71}$ observed a normal response 
RV end diastolic volume change

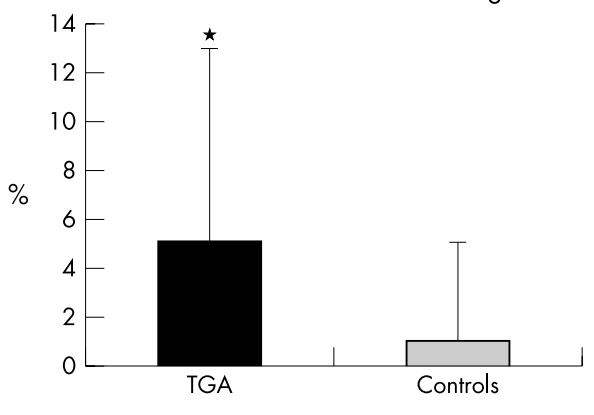

RV stroke volume change

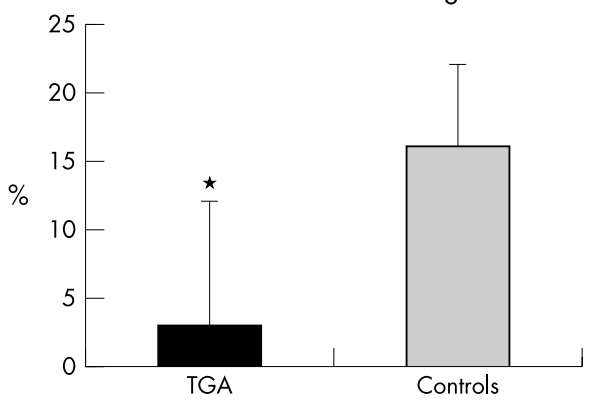

$\mathrm{RV}$ end systolic volume change

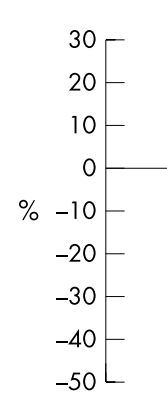

TGA

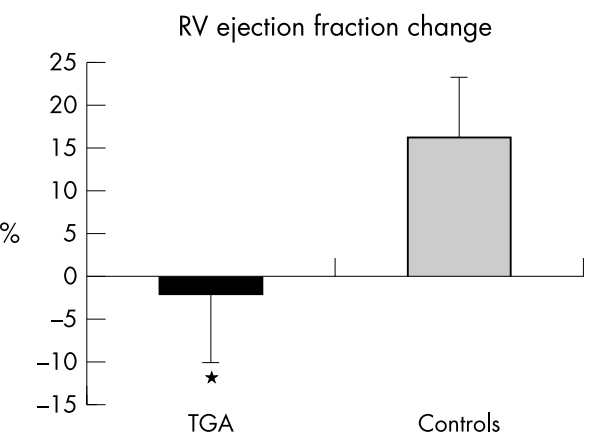

Figure 2 Percentage change of right ventricular (RV) volumes and function in response to exercise in patients with atrially corrected transposition of the great arteries (TGA) and healthy controls. The abnormal response of the systemic right ventricle of the TGA patients is shown by a significant increase in end diastolic volume and end systolic volume and no change in stroke volume and ejection fraction. *Significant difference in change between patients and controls. of right ventricular ejection fraction to exercise in the majority of patients after Mustard correction of TGA.

However, for optimal interpretation of changes in stroke volume and ejection faction in response to exercise, ventricular volumes should be quantified, which is difficult with techniques other than MRI. ${ }^{17}{ }^{19}$ Using MRI measurements, we observed an increase in right ventricular end diastolic volume and end systolic volume in response to exercise, whereas both volumes of the left ventricle did not change significantly in the patients. The normal ventricular response, as observed in the control group, is characterised by no change in biventricular end diastolic volume and a decrease in biventricular end systolic volume. ${ }^{16} 4243$ An increase in right ventricular end diastolic volume in response to exercise resembles the response seen in older healthy people ${ }^{42}$ in patients with corrected tetralogy of Fallot with important pulmonary regurgitation, ${ }^{15}$ and in patients with ischaemic heart disease. ${ }^{44} 45$ In patients with coronary heart disease, exercise induced myocardial ischaemia and myocardial scar contribute to an increase in left ventricular end diastolic volume in response to supine physical exercise. ${ }^{46-48}$ In patients after atrial repair of TGA fixed and reversible myocardial perfusion defects ${ }^{34} 49$ and decreased myocardial flow reserve of the systemic right ventricle ${ }^{50}$ are commonly found. Therefore, our findings of an increase in right ventricular end diastolic volume and end systolic volume in response to exercise may be the result of abnormalities in myocardial perfusion in response to stress.

After atrial correction of TGA the occurrence of perfusion defects during stress is related to the duration of follow up after correction. ${ }^{34}$ This may explain the contrasting findings of no change or even a decrease in end diastolic volume of the right ventricle observed six to seven years after atrial correction of $\mathrm{TGA}^{73}$ compared with our observations of an increase in end diastolic volume in response to exercise.

The lack of increase in stroke volume observed in patients with atrially corrected TGA has been attributed to abnormalities in contractility of the systemic right ventricle, ${ }^{71}$ restricted flow through the atrial baffles during exercise, ${ }^{2} 39$ and increased exercise systemic vascular resistance. ${ }^{52}$

Recently, Derrick and colleagues ${ }^{2}$ observed an appropriate systemic right ventricular myocardial contractile reserve in patients 18 years after atrial correction of TGA by using pressure-volume loop analysis at rest and during dobutamine stress. The authors ${ }^{2}$ hypothesised that restricted filling through the atrial baffles is the most important factor in causing the abnormal stroke volume and ejection fraction response to physical exercise. This was also suggested by Tulevski and colleagues, ${ }^{53}$ who used dobutamine stress in combination with MRI. Our observations of right ventricular volume response to exercise suggest a negative influence of ischaemia and myocardial scar on contractility of the systemic right ventricle. ${ }^{75154}$

Differences in stress protocol and measured parameters make direct comparison of studies difficult. Various stress protocols focus on different aspects of the cardiovascular response to stress. Furthermore, cardiac response to dobutamine or physical stress is dependent on the amount of dobutamine infused ${ }^{55}$ and the level of exercise, ${ }^{56}$ respectively. Besides differences in methods, discrepancy in age at correction and duration of follow up after correction limit a direct comparison of results of various studies. The combination of exercise MRI and pressure-volume loop analysis ${ }^{57}$ may provide a tool to elucidate further the mechanism of cardiac dysfunction in response to physical exercise.

\section{Exercise capacity and cardiac function}

Exercise capacity of the TGA patients was diminished, as shown by significantly lower oxygen uptake and workload at peak exercise. Decreased exercise capacity is often found in this patient group. ${ }^{51}$ In our study, in the TGA patients we observed a significant correlation between both exercise right ventricular ejection fraction and changes in right ventricular ejection fraction with exercise on the one hand and the indexed peak oxygen uptake on the other. No direct relation 
between exercise capacity and cardiac response to exercise has been reported so far. ${ }^{51}$

Ejection fraction is influenced by preload, contractility, afterload, and heart rate. As discussed in the previous section, abnormalities in preload ${ }^{29}$ and myocardial dysfunction ${ }^{74} 49$ are common findings in this patient group and may influence exercise capacity. The impaired chronotropic response to exercise observed in our patients may influence ventricular ejection fraction $^{58}$ and thus exercise capacity, ${ }^{5}$ although a direct relation between chronotropic impairment and exercise capacity has not been established..$^{58}$ Benson and colleagues $^{7}$ did not observe abnormalities in afterload response to exercise in TGA patients after atrial correction, suggesting that afterload may play a limited part in the abnormal response of the right ventricle to physical exercise.

\section{Study limitations}

We studied a selected group of patients after atrial correction of TGA. NYHA functional class was I or II and regular cardiac rhythm was present. However, this is the patient group at risk for cardiac failure and arrhythmias, which are both risk factors for cardiac death. ${ }^{10}$ Therefore, this patient group should be closely monitored to detect signs of cardiac failure and abnormalities of cardiac rhythm.

Tricuspid valve flow was not evaluated during exercise because of difficulties in assessing atrioventricular valve flow at high heart rates. Therefore, valuable information on the effect of exercise on tricuspid valve regurgitation and the potential correlation with exercise capacity was not available. The development of slice tracking techniques ${ }^{59}$ may overcome this problem in the future.

During the MRI exercise protocol, the images were acquired during short exercise breaks, which is less physiological than measurement during exercise. The use of real time MRI may allow the evaluation of cardiac function during exercise.

\section{Conclusion}

In TGA patients late after atrial correction who had near normal cardiac function at rest, the use of exercise MRI showed an abnormal response of both the systemic right and the left ventricles. Furthermore, abnormal cardiac response to exercise, but not at rest, correlated with diminished exercise capacity in this patient group. We conclude that exercise MRI is well suited to the evaluation of biventricular function at rest and in response to exercise and provides a tool for close monitoring of cardiovascular function in TGA patients after atrial correction, who are at risk for late death.

\section{ACKNOWLEDGEMENTS}

The technical assistance of Robert G M van Steijn (Department of Pulmonology, Leiden University Medical Centre, the Netherlands) is gratefully acknowledged.

\footnotetext{
Authors' affiliations

A A W Roest", W A Helbingt, Department of Paediatric Cardiology, Leiden University Medical Centre, Leiden, the Netherlands H J Lamb, P Kunz, A de Roos $\ddagger$, Department of Radiology, Leiden University Medical Centre

E E van der Wall $\ddagger$, H W Vliegen, Department of Cardiology, Leiden University Medical Centre

J G van den Aardweg, Department of Pulmonology, Leiden University Medical Centre

*Also the Departments of Radiology and Cardiology, Leiden University Medical Centre, and the Interuniversity Cardiology Institute of The Netherlands, Utrecht, Netherlands

†Also the Department of Paediatric Cardiology, Erasmus Medical Centre-Sophia's Children Hospital, Dr Molewaterplein 60, 3000 CB Rotterdam, Netherlands

$\ddagger$ Also the Interuniversity Cardiology Institute of The Netherlands
}

\section{REFERENCES}

1 Hoffman J. Incidence of congenital heart disease. I. Postnatal incidence. Pediatr Cardiol 1995; 16:103-13.

2 Derrick GP, Narang I, White PA, et al. Failure of stroke volume augmentation during exercise and dobutamine stress is unrelated to load-independent indexes of right ventricular performance after the Mustard operation. Circulation 2000;102:III154-9.

3 Haas $F$, Wottke $M$, Poppert $H$, et al. Long-term survival and functional followup in patients after the arterial switch operation. Ann Thorac Surg 1999:68:1692-7.

4 Williams WG, Trusler GA, Kirklin JW, et al. Early and late results of a protocol for simple transposition leading to an atrial switch (Mustard) repair. J Thorac Cardiovasc Surg 1988;95:717-26.

5 Paul MH, Wessel HU. Exercise studies in patients with transposition of the great arteries after atrial repair operations (Mustard/Senning): a review. Pediatr Cardiol 1999;20:49-55.

6 Lorenz CH, Walker ES, Graham TP, et al. Right ventricular performance and mass by use of cine MRI late after atrial repair of transposition of the great arteries. Circulation 1995;92:11233-9.

7 Benson LN, Bonet J, McLaughlin P, et al. Assessment of right ventricular function during supine bicycle exercise after Mustard's operation. Circulation 1982;65: 1052-9.

8 Sampson C, Kilner PJ, Hirsch R, et al. Venoatrial pathways after the Mustard operation for transposition of the great arteries: anatomic and functional MR imaging. Radiology 1994;193:211-7.

9 Clarkson PM, Barratt-Boyles BG, Neutze JM. Late dysrhythmias and disturbances of conduction following Mustard operation for complete transposition of the great arteries. Circulation 1976;53:519-24.

10 Gewillig M, Cullen S, Mertens B, et al. Risk factors for arrhythmia and death after Mustard operation for simple transposition of the great arteries. Circulation 1991;84:111187-92.

11 Helbing WA, Hansen B, Ottenkamp J, et al. Long-term results of atrial correction for transposition of the great arteries: comparison of Mustard and Senning operations. J Thorac Cardiovasc Surg 1994;108:363-72.

12 Gelatt M, Hamilton RM, McCrindle BW, et al. Arrhythmia and mortality after the Mustard procedure: a 30-year single-center experience. J Am Coll Cardiol 1997;29:194-201

13 Sarkar D, Bull C, Yates R, et al. Comparison of long-term outcomes of atrial repair of simple transposition with implications for a late arterial switch strategy. Circulation 1999;100:11176-81.

14 Gibbons RJ, Lee KL, Cobb FR, et al. Ejection fraction response to exercise in patients with chest pain, coronary artery disease and normal resting ventricular function. Circulation 1982;66:643-8.

15 Roest AA, de Roos A, Lamb HJ, et al. Tetralogy of Fallot: postoperative delayed recovery of left ventricular stroke volume after physical exercise assessment with fast MR imaging. Radiology 2003;226:278-84.

16 Mols $\mathbf{P}$, Huynh CH, Naeije N, et al. Volumetric response of right ventricle during progressive supine exercise in men. Am J Physiol 1991;261:751-4.

17 Beier J, Wellnhofer $\mathrm{E}$, Oswald $\mathrm{H}$, et al. Accuracy and precision of angiographic volumetry methods for left and right ventricle. Int J Cardiol 1996;53:179-88.

18 Hartnell GG, Notarianni M. MRI and echocardiography: how do they compare in adults? Semin Roentgenol 1998;33:252-61.

19 Helbing WA, Bosch HG, Maliepaard C, et al. Comparison of echocardiographic methods with magnetic resonance imaging for assessment of right ventricular function in children. Am J Cardiol 1995;76:589-94.

20 Pattynama PM, Lamb HJ, van der Velde EA, et al. Reproducibility of MRIderived measurements of right ventricular volumes and myocardial mass. Magn Reson Imaging 1995;13:53-63.

21 Chung KJ, Simpson IA, Glass RF, et al. Cine magnetic resonance imaging after surgical repair in patients with transposition of the great arteries. Circulation 1988;77:104-9.

22 Rees S, Somerville J, Warnes C, et al. Comparison of magnetic resonance imaging with echocardiography and radionuclide angiography in assessing cardiac function and anatomy following Mustard's operation for transposition of the great arteries. Am J Cardiol 1988;61:1316-22.

23 Roest AA, Kunz P, Lamb HJ, et al. Biventricular response to supine physical exercise in young adults assessed with ultrafast magnetic resonance imaging Am J Cardiol 2001;87:601-5.

24 Roest AA, Kunz P, Helbing WA, et al. Prolonged cardiac recovery from exercise in asymptomatic adults late after atrial correction of transposition of the great arteries: evaluation with magnetic resonance flow mapping Am J Cardiol 2001;88:1011-7.

25 Rebergen SA, Helbing WA, van der Wall EE, et al. MR velocity mapping of tricuspid flow in healthy children and in patients who have undergone Mustard or Senning repair. Radiology 1995;194:505-12.

26 Pedersen EM, Kozerke S, Ringgaard S, et al. Quantitative abdominal aortic flow measurements at controlled levels of ergometer exercise. Magn Reson Imaging 1999; 17:489-94.

27 Van der Geest RJ, Niezen RA, van der Wall EE, et al. Automated measurement of volume flow in the ascending aorta using MR velocity maps: evaluation of inter- and intraobserver variability in healthy volunteers. J Comput Assist Tomogr 1998;22:904-11.

28 van der Geest RJ, Buller VG, Jansen E, et al. Comparison between manual and semiautomated analysis of left ventricular volume parameters from shortaxis MR images. J Comput Assist Tomogr 1997;21:756-65.

29 Lorenz $\mathrm{CH}$, Walker ES, Morgan VL, et al. Normal human right and left ventricular mass, systolic function, and gender differences by cine magnetic resonance imaging. J Cardiovasc Magn Reson 1999;1:7-21. 
30 Lorenz $\mathrm{CH}$. The range of normal values of cardiovascular structures in infants, children, and adolescents measured by magnetic resonance imaging. Pediatr Cardiol 2000;21:37-46.

31 Berger HJ, Zaret BL. Nuclear cardiology (second of two parts). N Engl J Med 1981;305:855-65.

32 Clark AL, Poole-Wilson PA, Coats AJ. Effects of motivation of the patient on indices of exercise capacity in chronic heart failure. Br Heart $J$ 1994;71:162-5.

33 Wong KY, Venables AW, Kelly MJ, et al. Longitudinal study of ventricular function after the Mustard operation for transposition of the great arteries: a long term follow up. Br Heart J 1988;60:316-23.

34 Lubiszewska B, Gosiewska E, Hoffman P, et al. Myocardial perfusion and function of the systemic right ventricle in patients after atrial switch procedure for complete transposition: long-term follow-up. J Am Coll Cardiol 2000;36:1365-70.

35 Gatzoulis MA, Walters J, McLaughlin PR, et al. Late arrhythmia in adults with the Mustard procedure for transposition of great arteries: a surrogate marker for right ventricular dysfunction? Heart 2000;84:409-15.

36 Hochreiter C, Snyder MS, Borer JS, et al. Right and left ventricular performance 10 years after Mustard repair of transposition of the great arteries. Am J Cardiol 1994;74:478-82.

37 Murphy JH, Barlai-Kovach MM, Mathews RA, et al. Rest and exercise right and left ventricular function late after the Mustard operation: assessment by radionuclide ventriculography. Am J Cardiol 1983;51:1520-6.

38 Parrish MD, Graham TP, Bender HW, et al. Radionuclide angiographic evaluation of right and left ventricular function during exercise after repair of transposition of the great arteries: comparison with normal subjects and patients with congenitally corrected transposition. Circulation 1983;67:178-83.

39 Peterson RJ, Franch RH, Fajman WA, et al. Comparison of cardiac function in surgically corrected and congenitally corrected transposition of the great arteries. J Thorac Cardiovasc Surg 1988;96:227-36.

40 Ramsay JM, Venables AW, Kelly MJ, et al. Right and left ventricular function at rest and with exercise after the Mustard operation for transposition of the great arteries. Br Heart J 1984:51:364-70.

41 Hechter SJ, Webb G, Fredriksen PM, et al. Cardiopulmonary exercise performance in adult survivors of the Mustard procedure. Cardiol Young 2001;11:407-14.

42 Stratton JR, Levy WC, Cerqueira MD, et al. Cardiovascular responses to exercise: effects of aging and exercise training in healthy men. Circulation 1994:89: 1648-55.

43 Schaffer MS, de Souza M, Gilday DL, et al. Exercise radionuclide right ventriculography in children. Pediatr Cardiol 1987;8:235-40.

44 Newman GE, Rerych SK, Upton MT, et al. Comparison of electrocardiographic and left ventricular functional changes during exercise. Circulation 1980;62:1204-11

45 Manyari DE, Kostuk WJ. Left and right ventricular function at rest and during bicycle exercise in the supine and sitting positions in normal subjects and patients with coronary artery disease: assessment by radionuclide ventriculography. Am J Cardiol 1983;51:36-42.

46 Carroll JD, Hess OM, Hirzel HO, et al. Dynamics of left ventricular filling at rest and during exercise. Circulation 1983;68:59-67.

47 Manyari DE, Melendez $\sqcup$, Driedger AA, et al. Left ventricular function and volume during supine exercise in subjects with coronary artery disease. $J$ Appl Physiol 1981;50:636-42.

48 Mann DL, Scharf J, Ahnve S, et al. Left ventricular volume during supine exercise: importance of myocardial scar in patients with coronary heart disease. J Am Coll Cardiol 1987;9:26-34.

49 Millane T, Bernard EJ, Jaeggi E, et al. Role of ischemia and infarction in late right ventricular dysfunction after atrial repair of transposition of the great arteries. J Am Coll Cardiol 2000;35:1661-8.

50 Singh TP, Humes RA, Muzik O, et al. Myocardial flow reserve in patients with a systemic right ventricle after atrial switch repair. J Am Coll Cardiol 2001;37:2120-5.

51 Page $\mathrm{E}$, Perrault $\mathrm{H}$, Flore $\mathrm{P}$, et al. Cardiac output response to dynamic exercise after atrial switch repair for transposition of the great arteries. Am J Cardiol 1996;77:892-5.

52 Ensing GJ, Heise CT, Driscoll DJ. Cardiovascular response to exercise after the Mustard operation for simple and complex transposition of the great vessels. Am J Cardiol 1988;62:617-22.

53 Tulevski II, Lee PL, Groenink M, et al. Dobutamine-induced increase of right ventricular contractility without increased stroke volume in adolescent patients with transposition of the great arteries: evaluation with magnetic resonance imaging. Int J Card Imaging 2000;16:471-8.

54 Hornung TS, Kilner PJ, Davlouros PA, et al. Excessive right ventricular hypertrophic response in adults with the Mustard procedure for transposition of the great arteries. Am J Cardiol 2002;90:800-3.

55 Steendijk P, Baan J, van der Velde ET, et al. Effects of critical coronary stenosis on global systolic left ventricular function quantified by pressure-volume relations during dobutamine stress in the canine heart. $J$ Am Coll Cardiol 1998:32:816-26.

56 Braden DS, Carroll JF. Normative cardiovascular responses to exercise in children. Pediatr Cardiol 1999;20:4-10

57 Pattynama PM, de Roos A, van der Velde ET, et al. Magnetic resonance imaging analysis of left ventricular pressure-volume relations: validation with the conductance method at rest and during dobutamine stress. Magn Reson Med 1995; 34:728-37.

58 Paridon SM, Humes RA, Pinsky WW. The role of chronotropic impairment during exercise after the Mustard operation. J Am Coll Cardiol 1991:17:729-32.

59 Kozerke S, Scheidegger MB, Pedersen EM, et al. Heart motion adapted cine phase-contrast flow measurements through the aortic valve. Magn Reson Med 1999:42:970-8.

\section{FROM BMJ JOURNALS \\ A new form of retinopathy associated with myocardial infarction treated with percutaneous coronary intervention}

N Kinoshita, A Kakehashi, T Yasu, T Katayama, M Kuroki, Y Tsurimaki, R Ono, H Yamagami, M Saito, M Kawakami

Please visit the Heart website [www. heartinl.com] for a link to the full text of this article.
Aim: To report a new form of retinopathy that was observed in patients who had undergone percutaneous coronary intervention (PCI) following acute myocardial infarction (AMI).

Methods: Serial ophthalmological examinations were conducted in 40 patients who underwent PCI. Thirty patients were diagnosed with AMI, and another 10 had stable angina pectoris.

Results: Cotton wool spots developed in 17 (57\%) patients from the group with AMI undergoing PCI $(n=30)$ within 2 months. Of these, $41 \%$ (seven patients) also developed superficial haemorrhages. Retinopathy was most prominent 1-2 months after AMI and then tended to become quiescent afterwards, without treatment.

Conclusion: We have identified a new form of retinopathy in patients with AMI that spontaneously subsides without treatment.

A British Journal of Ophthalmology 2004;88:494-496. 